\title{
Urheberrecht an Bildschirmtext-Grafiken
}

\author{
LG Berlin, Urteil vom 6. Mai 1986 (16 O 72/86)
}

\section{Nichtamtlicher Leitsatz}

Bildschirmtext-Grafiken sind in der Regel urheberrechtlich nicht geschützt, sondern als rein handwerkliches Schaffen anzusehen, auch wenn die Definition von Grafikelementen in Bildpunktfelder eine schwierige und zeitaufwendige Tätigkeit darstellt.

\section{Paragraphen}

UnhG: §2; UWG: $\S 1$

\section{Stichworte}

Bildschirmtext-Grafiken - Urheberrechtsschutz unmittelbare Leistungsübernahme

\section{Tatbestand}

„Die Parteien sind Wettbewerber auf dem Gebiet der Btx-Dienstleistungen, wozu u.a. auch die Erstellung von Grafiken gehört. Sie bieten den Btx-Teilnehmern sogenannte Btx-Grafik-Grußkarten an, die von BtxTeilnehmern an andere Teilnehmer versendet werden können.

Die Klägerin behauptet: Sie habe ... ein Btx-Bild gestaltet. Dieses bestehe hauptsächlich aus der bildhaften Darstellung dreier Motive, nämlich einer Dorfkirche und zwei verschiedener Häusertypen ... Im Programm der Beklagten befänden sich Weihnachts- und Neujahrsgrußkarten, die zum Teil diese Motive enthalten hätten. So seien das Motiv Dorfkirche und die beiden Häusertypen zusammen mit anderen Grafikelementen dergestalt veröffentlicht worden, daß die Häuser mehrfach kopiert um die Kirche gruppiert und nur die Farben verändert worden seien.

Sie ist der Ansicht: Die von ihr gestalteten Grafikmotive genössen Urheberrechtsschutz ... Die Beklagte verstoße auch gegen die Vorschriften des lauteren Wettbewerbs. Der Kunde nehme an, daß das plagiierte Werk eine Eigenschöpfung der Beklagten sei. Sie werbe daher mit fremder Leistung für eigenes Können ... Den neben dem Unterlassungsanspruch geltend gemachten Schadensersatzanspruch sieht die Klägerin unter den Gesichtspunkten des entgangenen Gewinns und der Herstellungskosten als gerechtfertigt an."

\section{Entscheidungsgründe}

„Die Klage ist unbegründet. Der Klägerin steht unter keinem rechtlichen Gesichtspunkt ein Unterlassungsoder ein Schadensersatzanspruch zu:
1. Sie stützt ihren Anspruch zunächst auf $\S 97$ Abs. 1 UrhG. Die Voraussetzungen dieser Vorschrift sind jedoch nicht erfüllt, da es sich bei den Btx-Motiven, nämlich der Dorfkirche und den zwei verschiedenen Häusertypen, nicht um rechtlich geschützte Werke der bildenden Künste, insbesondere der angewandten Künste im Sinne von $\S 2$ Abs. 1 Ziff. 4 UrhG handelt. $\S 2$ Abs. 2 UrhG definiert den Begriff Werk als persönliche geistige Schöpfungen. Nach der ständigen Rechtsprechung des Bundesgerichtshofes ist unter Kunstwerk eine eigenpersönliche Schöpfung zu verstehen, die mit den Darstellungsmitteln der Kunst durch formgebende Tätigkeit hervorgebracht wird und vorzugsweise für die Anregung des ästhetischen Gefühls durch Anschauung bestimmt ist. Der ästhetische Gehalt muß jedoch einen solchen Grad erreicht haben, daß von einer künstlerischen Leistung gesprochen werden kann (Grundsatzurteil des Bundesgerichtshofs BGH GRUR 85, 289, 290 „Tonfiguren“).

Letztlich hängt der urheberrechtliche Werkcharakter davon ab, ob die zu beurteilenden Btx-Motive nicht nur eine geistige Schöpfung darstellen, sondern diese darüber hinaus eine individuelle Eigenart aufweisen. Erst ein Überragen der von durchschnittlich befähigten Gestaltern durch ,handwerkliche“ Tätigkeit geschaffenen Darstellungen durch eigenartige Prägung, individuelle Geistestätigkeit und einen erheblichen ästhetischen Überschuß über die Zweckform hinaus begründet die Urheberrechtsschlitzfähigkeit. Die rein handwerkliche Leistung, die jedermann mit durchschnittlichen Fähigkeiten ebenso zustande brächte, mag sie auch auf anerkennenswertem Fleiß und solidem Können beruhen, liegt außerhalb der Schutzfähigkeit (ständige Rechtsprechung; vgl. BGH GRUR 85, 1041, 1047 „Inkasso-Programm“; v. Gamm, Urheberrechtsgesetz, §2 Rdn. 16; Hubmann, Urheber- und Verlagsrecht, 5. Aufl. 1984, §15 I 1). Den von der Klägerin hergestellten Motiven, seien sie auch handwerklich durchaus gelungen und unter erheblichem Zeitaufwand hergestellt, haftet aber nicht die erforderliche individuelle Prägung an, die diese als Kunstwerk im Sinne von $\S 2$ Urheberrechtsgesetz qualifizieren würden ...

Es fehlt diesen Motiven an einer besonderen geistigen Konzeption der schöpferischen Gestältung, die über das hinausgeht, was im Bereich des Mediums Bildschirmtext normalerweise möglich erscheint. Den Grafikelementen liegen bekannte Darstellungen der betreffenden Gegenstände, die Allgemeingut sind, zugrunde, die lediglich in eine Bildschirm-Grafik übertragen wurden. Durch die begrenzte Anzahl von Bildpunkten je Zeichen sind hier bei der Gestaltung enge 
Grenzen gesetzt, die zwangsläufig zu einer bestimmten Abstraktion des Motivs führen. Die für die Schutzfähigkeit erforderliche Gestaltungshöhe, die gekennzeichnet ist durch ein bedeutendes schöpferisches Überragen rein handwerklicher Darstellungen, sei es durch spezifische Ausstrahlung, sei es aufgrund eigenartiger Prägung, aufgrund derer der Betrachter über das konkret Wahrgenommene hinaus einen besonderen ästhetischen Anstoß erfährt, ist in diesen Motiven nicht erkennbar (bgl. BGH GRUR 85, 289, 290 „Tonfiguren“; Hubmann, a.a.O., $\$ 15$ I) ... Die Definition von bekannten Grafikelementun in Bildpunktfelder ist keine schutzfähige Leistung, sondern, auch wenn es sich hierbei um eine schwierige und zeitaufwendige Tätigkeit handelt, als rein handwerkliches Schaffen anzusehen

2. Es bestehen auch keine wettbewerbsrechtlichen Ansprüche der Klägerin. Grundsätzdich kammt in engen Grenzen neben dem Werkschutz aufgrund des Urheberrechtsgesetzes noch ein Leistungsschutz gemäß $\S 1$ UWG in Betracht. Hierbei ist jedoch zu differenzieren, ob es sich um einen Fall der unmittelbaren Leistungsübernahme oder der Nachahmung handelt ... Es erscheint durchaus möglich, daß die Motive der Klägerin manuell durch Tastendruck überspielt werden können; die Grafik der Beklagten weist aber eine andere Farbgestaltung und Unterschiede in Details, wie z. B. die Position des Schornsteins, auf, so daß eine unmittelbare Leistungsübernahme nicht in Betracht kommt. Es handelt sich vielmehr um einen Fall der Nachahmung. §1 UWG hat aber nicht die Aufgabe, die nachgeahmte Leistung als solche zu schützen, selbst wenn sie mit erheblicher Mühe und großen Kosten geschaf- fen worden ist, da dies allein Zweck der Sondergesetze ist und andernfalls über $\S 1$ UWG ein weiterer, ergänzender Werkschutz eingeführt würde. Die Nachahmung nicht geschützter Werke ist daher in der Regel erlaubt und entspricht dem Interesse der Allgemeinheit, es sei denn, es treten besondere, außerhalb des kunstschutzrechtlichen Tatbestandes liegende Umstände hinzu, welche die Nachahmung wettbewerbsrechtlich als unlauter erscheinen lassen (BGHZ 5, 1, 10 „Hummel-Figuren“; Reimer/v. Gamm, Wettbewerbsrecht, Kap. 29 Rdn. 3, 8). Solche besonderen Umstände, die die Sittenwidrigkeit der Nachabınung der Motive durch die Beklagte zur Folge hätten, sind nicht ersichtlich ...".

\section{Anmerkung}

Dem Urteil ist zuzustimınen. Es liegt auf der Linie der urheberrechtlichen und wettbewerbsrechtlichen Rechtsprechung.

Btx-Grafiken können nur bei besonders herausragenden Leistungen Aussicht auf Anerkennnng als urheberrechtlich geschützte Werke haben. An der Rechtsprechung, die für die Zuerkennung des Usheberrechtsschutzes bei Grafiken sowie bei Computer-Programmen höhere Anforderungen stellt als bei Schriftwerken, ist festzuhalten. Denn die begrenzte Auflösung einer Bildschirmtext-Seite im CEPT-Standard hätte anderenfalls zur Folge, daß innerhalb kürzester Zeit kaum noch ein Anbieter das Risiko eingehen könnte, bestimmte häufig benutzte Motive aus dem Bereich alltäglicher Gegenstände in seiner grafischen Gestaltung zu benutzen.

Helmut Hoffmann

\section{Datenbanken}

\section{Volltext-Datenbanken und juristische Infor- mationssuche: Mit dem Rücken zur Zukunft}

(Teil 3 und Schluß)

\section{Robert C. Berring}

(Teil 2 :

II. Das neue Paradigma: Das Auftreten von LEXIS und WESTLAW

A. Die Entwicklung des Systems

B. Die Struktur des Systems: Volltext-Datenbanken und Freitextsuche

C. Stärken und Schwächen des Systems

1. Die mechanische Begrenzung des Systems: Die Effizienz der Freitextsuche

a. Das Wiederauftauchen des Themas: Die Studie von Blair und Maron)

Teil 3:

b. Der Fluch des Thamus: Man findet Worte, aber keine Weisheit c. Die Fehlerquote und der riesige Umfang der Datenbanken

2. Die Grenzen auf Seiten des Benutzers

a. Training von Inkompetenz ... oder schlimmer

b. Benutzer-Freundlich oder Benutzer-Verführend? - Der idiotensichere Cadillac

3. Einige theoretische Implikationen des neuen Paradigmas

III. Schlußfolgerung

A. Auf kurze Sicht: Computereinsatz als Ergänzung zur traditionellen Suche

B. Auf lange Sicht: Erweiterte computergestützte Suche statt traditioneller Suche 